\title{
NEW OBSERVATIONAL DATA CONCERNING \\ 4 HER AND $\zeta$ TAU
}

\author{
PETR HARMANEC, PAVEL KOUBSKÝ, JIŘí KRPATA, \\ and \\ FRANTIŠEK ŽĎÁRSKÝ \\ Astronomical Institute of the Czechoslovak Academy of Sciences, Ondřejov, Czechoslovakia
}

\begin{abstract}
Rectified intensity profiles of the $\mathrm{H} \alpha$ and $\mathrm{H} \beta$ Balmer lines of the shell star 4 Her were studied on 86 coudé spectrograms taken during 1969-1974. The central intensities of both lines vary periodically with the period of velocity changes equal to $\mathbf{4 6 . 1 9 4}$ days, reaching two maxima and minima each cycle. Radial-velocity curves of individual Balmer lines differ systematically one from another, the amplitude of the $\mathrm{H} \alpha$ variations being largest. The $V / R$ ratio of the $\mathrm{H} \alpha$ emission peaks varies in phase with the velocity changes. The velocity of the $\mathrm{H} \alpha$ emission is found to be almost invariable. A model is suggested to explain the observed variations in which $4 \mathrm{Her}$ is considered to be an interacting binary. The full paper will appear in Bull. Astron. Inst. Czech. 27, No. 1 in 1976.

Radial velocities of high-dispersion spectrograms of $\zeta$ Tau, obtained mostly during the last observing season, indicate the following facts: (1) Several distinct velocity systems are present in the Balmer lines, not a continuous progression. (2) The main absorption component of the $\mathrm{H} \alpha$ line does not share the long-term velocity variations, its velocity being always rather close to the systemic velocity $+22 \mathrm{~km} \mathrm{~s}^{-1}$. (3) The pseudoperiod of the long-term velocity variations has changed and is probably decreasing.
\end{abstract}

